\title{
Completeness of medication reviews provided by community pharmacists
}

\author{
H. F. Kwint*† PhD, A. Faber† PhD, J. Gussekloo ${ }^{*}$ MD PhD and M. L. Bouvy $†$ PhD \\ *Division of Pharmacoepidemiology E Clinical Pharmacology, Utrecht Institute for Pharmaceutical Sciences (UIPS), Utrecht, the Netherlands, $\dagger$ SIR Institute for \\ Pharmacy Practice and Policy, Leiden, the Netherlands and $\ddagger$ Department of Public Health and Primary Care, Leiden University Medical Center, Leiden, the \\ Netherlands
}

Received 9 July 2013, Accepted 16 December 2013

Keywords: community pharmacists, drug-related problems, expert reviewers, medication review

\section{SUMMARY}

What is known and objectives: Little is known about the ability of community pharmacists who are inexperienced in medication review to identify drug-related problems (DRPs). The objective of our study was to investigate the completeness of DRPs in terms of number, type and clinical relevance identified by community pharmacists when performing home medication reviews (HMRs).

Methods: This is a cross-sectional study within the intervention arm of a randomized controlled trial among communitydwelling patients ( $\geq 65$ years, $\geq 5$ drugs) in ten Dutch community pharmacies. Community pharmacists, who were inexperienced in medication review, received 2-day training in medication review. These pharmacists interviewed patients at home about their medicines, identified potential DRPs and made recommendations in combination with medication and clinical records. Expert reviewers completed the number of potential DRPs and recommendations by reviewing all available information, including patient interview reports.

Results and discussion: In 155 patients, community pharmacists identified a mean of 3.6 (SD 2.8) potential DRPs per patient and expert reviewers added 6.5 (SD 3.2) DRPs. Community pharmacists formulated 2.6 (SD 2.3) recommendations per patient and reviewers added 7.5 (SD 3.3) recommendations. Community pharmacists identified a higher proportion of clinically relevant DRPs compared with expert reviewers, as assessed by DRPs with high priority $[O R=1 \cdot 8(95 \%$ CI 1.4-2.2)], DRPs associated with recommendations for drug change $[\mathrm{OR}=1.9(95 \%$ CI $1.5-$ 2.3)] and implemented recommendations for drug change [OR $=2 \cdot 1(95 \%$ CI 1.6-2.7)].

What is new and conclusion: This study shows that the completeness of medication reviews by inexperienced community pharmacists with limited training could be improved, although they identified a higher proportion of potentially clinically relevant DRPs compared with expert reviewers. The results suggest that community pharmacists with limited experience in medication review may need more intensive post-graduate training.

\section{WHAT IS KNOWN AND OBJECTIVES}

Several randomized controlled trials (RCTs) have demonstrated that clinical medication review can resolve drug-related problems

Correspondence: H. F. Kwint, Division of Pharmacoepidemiology \& Clinical Pharmacology, Utrecht Institute for Pharmaceutical Sciences (UIPS), Utrecht, the Netherlands. Tel.: +0031715766311; fax: +0031715766251; e-mail: h.f.kwint@apotheekstevenshof.nl
(DRPs). However, these RCTs differed in the expertise of participating pharmacists who were either highly trained ${ }^{1-10}$ or inexperienced in medication review. ${ }^{11-13}$ Medication review is a complex intervention, which requires both knowledge and skills. ${ }^{14,15}$ Medication review skills have to be developed to explore patient's experiences and beliefs about medicines, to identify potential drug-related problems (DRPs), formulate recommendations for a pharmaceutical care plan and to discuss this care plan with physicians. ${ }^{15}$

No extensive post-graduate courses in medication review were available at the start of this RCT on home medication review (HMR) in the Netherlands. ${ }^{16}$ Therefore, community pharmacists, who were inexperienced in medication review, received a two-day training course as a part of this study where pharmacists were taught on pharmacotherapy and medication review skills. As part of the intervention, pharmaceutical care plans by the community pharmacists were evaluated by independent expert reviewers. ${ }^{16}$

Relatively little is known to what extent community pharmacists are able to identify DRPs in medication review. ${ }^{17,18}$ In particular, no research has been performed into the clinical relevance of these DRPs. The objective of this study was to investigate the completeness of DRPs in terms of number, type and clinical relevance identified by community pharmacists in HMRs.

\section{METHODS}

\section{Study design}

This is a cross-sectional study among 155 community-dwelling patients ( $\geq 65$ years and $\geq 5$ drugs including at least one cardiovascular or one antidiabetic drug) enrolled in the intervention arm of an RCT on HMRs in ten community pharmacies.

\section{Intervention}

Community pharmacists who were inexperienced in medication review received 2 days of additional training. Pharmacists had access to the patient's medication records from the pharmacy, which are generally a comprehensive source of information as the majority of patients in the Netherlands are registered at only one community pharmacy, independently of prescriber. ${ }^{19}$ Medical history and laboratory data of the patient were collected with the help of the GP practice. As part of the study protocol, patients were offered additional laboratory measurements of $\mathrm{HbA} 1_{c}$ cholesterol, sodium, potassium and creatinine and blood pressure measurement. Pharmacists interviewed patients at home about their medicines, identified potential DRPs and made recommendations in pharmaceutical care plans. Subsequently, expert 
reviewers (A.F., J.K.-D. and H.K.) completed the number of potential DRPs and recommendations by reviewing all available information, including patient interview reports. Expert reviewers had several years of experience with medication review as well as in-depth knowledge of national clinical guidelines. More details about the intervention have been described elsewhere. ${ }^{16}$

\section{Data collection and classification}

Data extracted from the completed pharmaceutical care plans included medication and medical information, patient interview reports, identified potential DRPs and associated recommendations as well as the prioritization for implementation (high, medium or low). Complete patient medication records from the community pharmacy including drug-dispensing records until at least 6 months after the patient interview were collected separately.

Each potential DRP and recommendation were classified as being identified by community pharmacist or added during completion by pharmacist reviewer. When the description of a DRP or recommendation was incomplete and refined by a pharmacist reviewer, this DRP was assigned to the community pharmacist. All potential DRPs and associated recommendations were classified using the D.O.C.U.M.E.N.T. classification system using the most recent version. ${ }^{16,20,21}$ Clinical relevance was assessed by the percentage of DRPs assigned a high priority, the percentage of recommendations for drug change and the percentage of implemented recommendations for drug change. Implemented recommendations for drug change were assessed by analysing drug-dispensing records for medication changes.

All coding and classification was independently undertaken by one investigator (H.K.) and a student investigator (Y.A.). When there were differences in coding, the investigators reached consensus in a case conference with a third investigator (A.F. or M.B.).

\section{Statistical analysis}

Pearson chi-squared tests were used to compare categorical variables. Independent $t$-tests were used to compare the mean number of DRPs per patient. Differences between the percentages of clinically relevant DRPs and recommendations identified by community pharmacists and those added by expert reviewers were compared by odds ratios (ORs) and corresponding 95\% confidence intervals.

A $P$-value $<0.05$ was considered statistically significant. All data were analysed using Microsoft Access 2007 (Microsoft Corporation, Redmond, WA, USA) and SPSS version 17.0 (SPSS Inc., Chicago, IL, USA).

\section{RESULTS}

\section{Identified DRPs and recommendations}

During the study period, 16 different community pharmacists in 10 community pharmacies were involved. Community pharmacists identified 553 potential DRPs (mean 3.6 , SD 2.8 per patient) and expert reviewers 1012 potential DRPs (mean 6.5, SD 3.2 per patient; Table 1). Community pharmacists formulated 398 recommendations (mean 2.6, SD $2 \cdot 3$ per patient) and expert reviewers 1167 recommendations (mean 7.5 , SD 3.3 per patient, $P<0.01$; Table 2). Considerable variations were observed in the proportion of DRPs identified by community pharmacists (range 13-57\%) and recommendations formulated by community pharmacists (range $10-46 \%)$.

All DRP types, except 'Compliance', were in absolute numbers more frequently added by expert reviewers. DRP types 'Toxicity' $(P<0.01)$ and 'Undertreated' $(P=0.04)$ were relatively more frequently identified by community pharmacists (Table 1 ).

All recommendations were in absolute numbers more frequently added by expert reviewers, except 'Drug formulation change'. 'Addition of drug' was relatively more frequently recommended by community pharmacists $(P=0.02)$ (Table 2$)$.

\section{DRPs identified in patient interviews}

Of 415 potential DRPs originating from patient interviews, community pharmacists identified 171 DRPs (mean 1.1 per patient, SD 1.1) and expert reviewers added 244 DRPs (mean 1.6 per patient, SD 1.7) $(P<0.01)$. 'No indication apparent' $(n=64,26 \%)$, 'Condition undertreated' ( $n=33,14 \%)$, 'Toxicity, allergic reaction or adverse effect present' $(n=30,12 \%)$ and 'Incorrect or unclear dosing instructions' $(n=29,12 \%)$ were the main DRP subtypes added by expert reviewers from patient interviews.

\section{Clinical relevance}

Community pharmacist identified a higher proportion of DRPs with a high priority compared with expert reviewers [OR 1.8 (1.4-2.2), $P<0 \cdot 01]$. Furthermore, a higher proportion of DRPs followed by recommendations for a drug change was identified by community pharmacists compared with expert reviewers [OR 1.9 (1.5-2.3), $P<0 \cdot 01]$. Finally, a higher proportion of DRPs followed by implemented recommendations for drug change was identified by community pharmacists [OR $2 \cdot 1(1 \cdot 6-2 \cdot 7), P<0 \cdot 01$ ] (Table 3$)$.

\section{DISCUSSION}

This study shows that expert reviewers identified considerably more potential DRPs and associated recommendations than community pharmacists. However, the DRPs identified by community pharmacists were more often clinically relevant compared with DRPs added by expert reviewers.

Expert reviewers almost doubled the amount of DRPs identified by community pharmacists. The highest difference was seen for the DRP type 'Monitoring'. Reviewers especially added DRPs related to appropriate monitoring of hypertension, dyslipidaemia, diabetes $^{22,23}$ and other diseases. Community pharmacists rarely identified these. Monitoring-related problems accounted for more than two DRPs per patient, but were mostly assigned a low priority and not followed by a recommendation for drug change. Monitoring-related problems and some other DRPs identified by expert reviewers might be perceived by community pharmacists and GPs as too theoretical or 'textbook advice'. ${ }^{24}$ This may partly explain why community pharmacists identified a lower number but relatively more clinically relevant DRPs. Furthermore, community pharmacists may have ignored other potential DRPs, expecting that associated recommendations might not be implemented based on their earlier experiences with GPs and patients.

There was a considerable variation in the completeness of the reviews. We were not able to identify explicit indicators that may account for this variation in identified DRPs among pharmacists. All participating community pharmacists had no previous hospital experience, no additional clinical pharmacy skills nor followed 
Table 1. Comparison of number and type of drug-related problems (DRPs) identified by community pharmacists and added by expert reviewers for 155 patients

\begin{tabular}{|c|c|c|c|c|c|}
\hline \multirow[b]{2}{*}{ DRP type and subtype } & \multicolumn{2}{|c|}{ Community pharmacists } & \multicolumn{2}{|c|}{ Expert reviewers } & \multirow{2}{*}{$\begin{array}{l}P \text { - } \\
\text { value }\end{array}$} \\
\hline & $N$ & $\%^{\mathrm{a}}$ & $N$ & $\%^{\mathrm{a}}$ & \\
\hline Overall & 553 & 36 & 1012 & 64 & - \\
\hline Mean per patient $\pm S D$ & $3 \cdot 6$ & $\pm 2 \cdot 8$ & $6 \cdot 5$ & $\pm 3 \cdot 2$ & - \\
\hline $\mathbf{D}$ (rug selection) & 156 & 36 & 279 & 64 & 0.79 \\
\hline Duplication & 7 & 58 & 5 & 42 & 0.09 \\
\hline Drug interaction & 5 & 33 & 10 & 67 & $0 \cdot 87$ \\
\hline Contraindications apparent & 28 & 38 & 46 & 62 & 0.65 \\
\hline No indication apparent & 106 & 33 & 217 & 67 & 0.29 \\
\hline Other drug selection problem & 10 & 91 & 1 & 9 & $<0.01$ \\
\hline $\mathbf{O}($ ver or underdose $)$ & 64 & 38 & 105 & 62 & 0.47 \\
\hline Prescribed dosage too high & 14 & 42 & 19 & 58 & 0.39 \\
\hline Prescribed dosage too low & 31 & 43 & 41 & 57 & $0 \cdot 16$ \\
\hline Incorrect or unclear dosing instructions & 19 & 30 & 45 & 70 & 0.33 \\
\hline C(ompliance) & 52 & 54 & 44 & 46 & $<0.01$ \\
\hline Taking too little & 25 & 58 & 18 & 42 & $<0.01$ \\
\hline Taking too much & 5 & 63 & 3 & 38 & 0.11 \\
\hline Difficulty using dosage form & 22 & 49 & 23 & 51 & 0.05 \\
\hline $\mathbf{U}($ ndertreated $)$ & 159 & 40 & 243 & 60 & 0.04 \\
\hline Condition undertreated & 117 & 40 & 172 & 60 & 0.04 \\
\hline Condition untreated & 28 & 36 & 49 & 64 & 0.85 \\
\hline Preventive therapy required & 14 & 39 & 22 & 61 & $0 \cdot 65$ \\
\hline $\mathbf{M}$ (onitoring) & 75 & 21 & 284 & 79 & $<0.01$ \\
\hline Laboratory monitoring & 58 & 22 & 203 & 78 & $<0.01$ \\
\hline Non-laboratory monitoring & 17 & 17 & 81 & 83 & $<0.01$ \\
\hline $\mathbf{E}$ (ducation) or Information & 1 & 11 & 8 & 89 & $0 \cdot 13$ \\
\hline Disease management or advice & 1 & 11 & 8 & 89 & 0.13 \\
\hline $\mathbf{T}$ (oxicity) & 46 & 48 & 49 & 52 & $<0.01$ \\
\hline Toxicity, allergic reaction or adverse effect present & 46 & 48 & 49 & 52 & $<0.01$ \\
\hline
\end{tabular}

DRPs, drug-related problems; SD, standard deviation.

${ }^{a} \%$ is the percentage within type or subtype of recommendation.

earlier courses in medication review. Nevertheless, differences in knowledge of pharmacotherapy and medication review skills ${ }^{14,15}$ among community pharmacists were observed by expert reviewers. Furthermore, some pharmacists may have relied on the expert reviewers to complete the medication reviews. This may partly be explained by the fact that the registration of all research data was experienced as time-consuming by the participating pharmacists.

Surprisingly, a considerable part of issues that were discussed with the patients were not formally identified as potential DRP by community pharmacists. The most frequently added subtype of DRP by expert reviewers from patient interviews was 'Indication not apparent'. This refers to patients using drugs without knowing the indication, which was also lacking in the GP record, or refers to patients using drugs not intended for prolonged use. 'Condition undertreated' was also frequently added by reviewers (e.g. patients indicated that their pain treatment was suboptimal, whereas community pharmacists had not suggested a change of drug or dosing regimen). Furthermore, adverse effects were frequently described in patient interview reports, but surprisingly not always identified as potential DRPs. Possibly, community pharmacists did not recognize complaints as caused by adverse effects or did recognize these, but considered these as inevitable. Finally, 'Incorrect or unclear dosing instructions' was also frequently 'missed' as potential DRPs by community pharmacists. Community pharmacists may consider these medication manage- ment problems (e.g. time of intake) as typical pharmacist issues and of minor interest for discussion with GP. Often, these issues were directly solved during the patient interview by advice (e.g. change time of intake).

The percentage of identified DRPs by community pharmacists in our study $(36 \%)$ was comparable to findings of Krska and Avery $^{17}(34 \%)$ with a clinical pharmacist and an experienced GP as expert reviewers. In the study of Krska, training of the community pharmacists was limited to 2 days, specifically designed for the study, and no formal assessment of their competency to conduct medication reviews was made. ${ }^{17}$ Laaksonen et al. ${ }^{18}$ investigated the performance of community pharmacists who completed a more intensive post-graduate course (i.e. five 60-h distance learning modules in clinical therapeutics). These trained community pharmacists managed to identify $75 \%$ of the DRPs found by a clinical pharmacist. These comparisons with our study suggest that 2-day training in medication review may be too limited. A practical solution could be to enrol pharmacists inexperienced in medication review in an intensive post-graduate course in which feedback on reviews from expert reviewers (and portfolio building) plays an essential role.

This study had several strengths. First, a very detailed and accurate description of DRPs was available, because community pharmacists sent all pharmaceutical care plans to the expert reviewers. This enabled us to distinguish very clearly between 
Table 2. Comparisons of number and type of recommendations identified by community pharmacists and added by expert reviewers for 155 patients

\begin{tabular}{|c|c|c|c|c|c|}
\hline \multirow[b]{2}{*}{ Type of recommendation } & \multicolumn{2}{|c|}{ Community Pharmacists } & \multicolumn{2}{|c|}{ Expert Reviewers } & \multirow[b]{2}{*}{$P$-value } \\
\hline & $N$ & $\%^{\mathrm{a}}$ & $N$ & $\%^{\mathrm{a}}$ & \\
\hline Overall & 398 & 25 & 1167 & 75 & - \\
\hline Mean per patient \pm SD & 2.5 & $\pm 2 \cdot 3$ & 7.5 & $\pm 3 \cdot 3$ & - \\
\hline Recommendation for drug change & 272 & 30 & 633 & 70 & $<0.01$ \\
\hline Cessation of drug & 59 & 29 & 146 & 71 & $0 \cdot 24$ \\
\hline Dose increase & 31 & 32 & 67 & 68 & $0 \cdot 15$ \\
\hline Dose decrease & 16 & 25 & 47 & 75 & 0.99 \\
\hline Addition of drug & 85 & 31 & 190 & 69 & 0.02 \\
\hline Replacement of drug & 49 & 30 & 115 & 70 & $0 \cdot 17$ \\
\hline Dose frequency/schedule change & 20 & 27 & 54 & 73 & 0.75 \\
\hline Drug formulation change & 12 & 50 & 12 & 50 & $<0.01$ \\
\hline Recommended dose administration aid & 0 & 0 & 3 & 100 & 0.31 \\
\hline Other recommendations & 126 & 19 & 534 & 81 & $<0.01$ \\
\hline Education/counselling session & 10 & 20 & 39 & 80 & 0.41 \\
\hline Monitoring: Non-laboratory & 14 & 14 & 85 & 86 & $<0.01$ \\
\hline Monitoring: Laboratory & 59 & 20 & 232 & 80 & 0.03 \\
\hline Adjustment of patient records & 38 & 19 & 158 & 81 & 0.04 \\
\hline Other & 4 & 17 & 19 & 83 & 0.37 \\
\hline
\end{tabular}

$\mathrm{SD}$, standard deviation.

$\mathrm{a}_{\%}$ is the percentage within type or subtype of recommendation

Table 3. Comparison of clinical relevance of drug-related problems (DRPs) identified by community pharmacists and added by expert reviewers

\begin{tabular}{|c|c|c|c|c|c|c|}
\hline \multirow[b]{2}{*}{ Clinical relevance of DRPs } & \multicolumn{2}{|c|}{$\begin{array}{l}\text { Community } \\
\text { Pharmacists }\end{array}$} & \multicolumn{2}{|c|}{ Expert Reviewers } & \multirow[b]{2}{*}{ OR $(95 \% \mathrm{CI})$} & \multirow[b]{2}{*}{$P$-value } \\
\hline & $N$ & $\%$ & $N$ & $\%$ & & \\
\hline Overall (reference) & 553 & 100 & 1012 & 100 & Ref & - \\
\hline With high priority & 285 & 52 & 379 & 37 & $1.8(1.4-2 \cdot 2)$ & $<0.01$ \\
\hline With recommendations for drug change & 375 & 68 & 530 & 52 & $1.9(1.5-2 \cdot 3)$ & $<0.01$ \\
\hline With implemented recommendations for drug change & 132 & 24 & 133 & 13 & $2 \cdot 1(1 \cdot 6-2 \cdot 7)$ & $<0.01$ \\
\hline
\end{tabular}

OR, odds ratio; 95\% CI, 95\% confidence interval.

DRPs identified by community pharmacists and expert reviewers and also provided insight into the clinical relevance of DRPs. Second, the availability of detailed patient interview reports enabled us to assign DRPs originating from patient interviews.

There are some limitations associated with this study. First, expert reviewers in this study could only refine or add to the DRPs already identified by community pharmacists. Blinding of the reviewers for DRPs identified by community pharmacists would have enabled a more in-depth comparison between community pharmacists and expert reviewers. However, assuming that expert reviewers would have identified the majority of DRPs detected by community pharmacists, this would not have a major impact on the findings of the study. Second, expert reviewers were dependent on the provided documentation by community pharmacists for identifying DRPs and did not conduct patient interviews. On the one hand, expert reviewers at distance might have identified some potential DRPs in this study that they possibly might have neglected if they knew the patient. On the other hand, expert reviewers might have identified more or other DRPs from patient interviews. Third, the number of DRPs identified by community pharmacists may have been underestimated, as a limited number of issues were directly solved during the patient interview, but not registered in the pharmaceutical care plan by the pharmacists.

\section{WHAT IS NEW AND CONCLUSION}

This study shows that the completeness of medication reviews by inexperienced community pharmacists with limited training could be improved, although they identified a higher proportion of potentially clinically relevant DRPs compared with expert review- 
ers. This suggests that a 2-day training programme in medication review may be too limited.

\section{ACKNOWLEDGEMENTS}

The study received unrestricted research funding by the Royal Dutch Association of Pharmacists (KNMP), Astra-Zeneca and the healthcare insurance companies Achmea and Menzis. This work was performed totally independently from these funders. This study was conducted in pharmacies of BENU Apotheken (for- merly known as LLOYDS Apotheken). The authors would like to thank all participating community pharmacists, L.A. Schul of BENU Apotheken for her coordinating activities, J.M. KrijgerDijkema as a pharmacist reviewer and Y. Amarouchi, student, for his contribution to the data classification.

\section{CONFLICT OF INTERESTS}

All authors declare that they have no conflict of interests that are directly relevant to the content of this study.

\section{REFERENCES}

1. Bryant LJ, Coster G, Gamble GD, McCormick RN. The General Practitioner-Pharmacist Collaboration (GPPC) study: a randomised controlled trial of clinical medication reviews in community pharmacy. Int J Pharm Pract, 2011;19:94-105.

2. Grymonpre RE, Williamson DA, Montgomery PR. Impact of a pharmaceutical care model for non-institutionalised elderly: Results of a randomised, controlled trial. Int J Pharm Pract, 2001;9:235-241.

3. Krska J, Cromarty JA, Arris F et al. Pharmacist-led medication review in patients over 65: a randomized, controlled trial in primary care. Age Ageing, 2001;30:205-211.

4. Lenaghan E, Holland R, Brooks A. Homebased medication review in a high risk elderly population in primary care - The POLYMED randomised controlled trial. Age Ageing, 2007;36:292-297.

5. Sellors J, Kaczorowski J, Sellors C et al. A randomized controlled trial of a pharmacist consultation program for family physicians and their elderly patients. CMAJ, 2003;169:17-22.

6. Sorensen L, Stokes JA, Purdie DM, Woodward M, Elliott R, Roberts MS. Medication reviews in the community: results of a randomized, controlled effectiveness trial. Br J Clin Pharmacol, 2004;58:648-664.

7. Volume CI, Farris KB, Kassam R, Cox CE, Cave A. Pharmaceutical care research and education project: patient outcomes. $\mathrm{J} \mathrm{Am}$ Pharm Assoc, 2001;41:411-420.

8. Kassam R, Farris KB, Burback L, Volume CI, Cox CE, Cave A. Pharmaceutical care research and education project: pharmacists' interventions. J Am Pharm Assoc, 2001;41:401-410.

9. Williams ME, Pulliam CC, Hunter R et al. The short-term effect of interdisciplinary medication review on function and cost in ambulatory elderly people. J Am Geriatr Soc, 2004;52:93-98.

10. Zermansky AG, Petty DR, Raynor DK, Lowe CJ, Freemantle N, Vail A. Clinical medication review by a pharmacist of patients on repeat prescriptions in general practice: a randomised controlled trial. Health Technol Assess, 2002;6:1-86.

11. Bernsten C, Bjorkman I, Caramona $\mathrm{M}$ et al. Improving the well-being of elderly patients via community pharmacy-based provision of pharmaceutical care: a multicentre study in seven European countries. Drugs Aging, 2001;18:63-77.

12. Denneboom W, Dautzenberg MG, Grol $R$, De Smet PA. Treatment reviews of older people on polypharmacy in primary care: cluster controlled trial comparing two approaches. Br J Gen Pract, 2007;57:723731.

13. Bond C. The MEDMAN study: A randomized controlled trial of community pharmacy-led medicines management for patients with coronary heart disease. Fam Pract, 2007;24:189-200.

14. Laaksonen R, Mills ER, Duggan C, Davies JG, Bates M, Mackie CA. The effect of training and service provision on the self-assessed competence of community pharmacists. Int J Pharm Pract 2007;15:141-147.

15. Niquille A, Lattman C, Bugnon O. Medication reviews led by community pharmacists in Switzerland: a qualitative survey to evaluate barriers and facilitators. Pharm Pract, 2010;8:35-42.

16. Kwint HF, Faber A, Gussekloo J, Bouvy ML. The contribution of patient interviews to the identification of drug-related problems in home medication review. J Clin Pharm Ther, 2012;37:674-680.

17. Krska J, Avery AJ, Community Pharmacy Medicines Management Project Evaluation
Team. Evaluation of medication reviews conducted by community pharmacists: a quantitative analysis of documented issues and recommendations. Br J Clin Pharmacol, 2008;65:386-396.

18. Laaksonen R, Duggan C, Bates I. Performance of community pharmacists in providing clinical medication reviews. Ann Pharmacother, 2010;44:1181-1190.

19. Buurma H, Bouvy ML, De Smet PA, FloorSchreudering A, Leufkens HG, Egberts AC. Prevalence and determinants of pharmacy shopping behaviour. J Clin Pharm Ther, 2008;33:17-23.

20. Williams M, Peterson GM, Tenni PC, Bindoff IK, Stafford AC. DOCUMENT: a system for classifying drug-related problems in community pharmacy. Int J Clin Pharm, 2011;34:43-52.

21. Williams M, Peterson GM, Tenni PC et al. Drug-related problems detected in Australian Community Pharmacies: The PROMISe Trial. Ann Pharmacother, 2011;45:1067-1076.

22. Smulders YM, Burgers JS, Scheltens T, van Hout BA, Wiersma T, Simoons ML. Clinical practice guideline for cardiovascular risk management in the Netherlands. Neth J Med, 2008;66:169-174.

23. Bouma M, Rutten GE, de Grauw WJ, Wiersma T, Goudswaard AN, Nederlands Huisartsen Genootschap. Summary of the practice guideline 'Diabetes mellitus type 2' (second revision) from the Dutch College of General Practitioners. Ned Tijdschr Geneeskd, 2006;150:2251-2256.

24. Bryant L, Coster G, McCormick R. General practitioner perceptions of clinical medication reviews undertaken by community pharmacists. I Prim Health Care, 2010;2:225-233. 